\section{Challenges and limitations of targeting cancer stem cells and/or the tumor microenvironment}

\author{
Juan Sebastian Yakisich \\ Department of Clinical Neuroscience R54, \\ Karolinska University Hospital, \\ Karolinska Institute, Stockholm, Sweden
}

\begin{abstract}
The existence of cancer cells with stem cell properties (Cancer Stem Cells, CSCs) and their association with tumor resistance and relapse has led to the search for active compounds to eliminate these cells or modulate their stemness in the hope of curing cancer So far, three classes of drugs that target cancer stemness (Stemness Modulator Drugs) have been identified: i) drugs that selectively eliminate CSCs (stem cell targeting drugs); ii) drugs that decrease stemness (stemness inhibitor drugs); and iii) drugs that promote stemness (stemness promoting drugs). In addition, microenvironment modulating drugs aimed at selectively targeting the stem cell niche are being investigated and may represent an important class of drug for cancer therapy. This article will briefly review the current use of these substances and discuss the potential outcomes, challenges and limitations of treatment modalities using these classes of drugs for cancer treatment. Finally, a modular tumor model will be proposed as a guide to integrate our knowledge on the biology of cancer stem cell with that of the tumor microenvironment to promote a more rational development of anticancer therapy.
\end{abstract}

\section{Introduction}

In most types of cancers, different subpopulations of cancer stem cell (CSCs) or cancer stem-like cells (CS-LCs) have been described. There is a still considerable debate on the existence of CSCs and on the exact nature of these cells. ${ }^{1-4}$ Due to technical limitations and the lack of reliable markers for CSCs, the data from some scientific articles that reported the isolation of CSCs and their characterization in term of their biological properties and chemosensitivity should be taken with caution. To illustrate this, the initial identification of cancer stem cells in human brain tumors was made by assessing the expression of the neural stem cell surface marker CD133. ${ }^{5}$ In that study, CD133+ cells, but not
CD133- cells, showed marked stem cell activity. Later, several authors used CD133 as a glioma stem cell marker., ${ }^{6,7}$ In one study, $\mathrm{CD} 133^{+}$cells were significantly resistant to temozolomide, carboplatin, paclitaxel and etoposide compared to autologous CD133cells. ${ }^{7}$ In another study, temozolomide was shown to preferentially deplete $\mathrm{CD} 133^{+}$cells. $^{8}$ It was later found that CD133 is not a reliable marker for glioma stem cells since CD133glioblastoma cells (for the anti-CD133 antibody) actually express a truncated variant of the CD133 protein and that CD133- cells were also tumorigenic and able to repopulate the CD133+ fraction..$^{9,10}$ The limitations of CD133 as stem cell marker are not restricted to brain tumors ${ }^{11}$ and have also been documented for lung cancer. ${ }^{12}$ For some types of immunotherapy, CD133 status seems to be irrelevant since both $\mathrm{CD}_{133^{+}}$and $\mathrm{CD} 133^{-}$are susceptible to NK-mediated cytotoxicity. ${ }^{13}$ In consideration of these data, the real impact of the research performed on glioma stem cells using CD133 as a surface marker has to be carefully evaluated. Other methods to isolate cancer stem cells (e.g. side population (SP) fraction, neurospheres) also have severe limitations and, once again, the results should be analyzed with care. Due to space limitations, this issue will remain beyond the scope of this manuscript. In this manuscript, CSCs and CS-LCs will be used indistinctly and referred to as CSCs/CS-LCs.

The classical division between CSCs/CSLCs and non-CSCs is not the best model for cancer biology. Instead, there is a growing consensus that tumors are more heterogeneous and contain several subpopulations of cells with different degrees of stemness (CSCs/CS-LCs) that are usually associated with increased resistance to chemotherapy. ${ }^{14,15}$ It is not a surprise then that CSCs/CSLCs can be selected in vitro following treatment with anticancer drugs ${ }^{16,17}$ and standard chemotherapy enrich for CSCs/CS-LCs in both xenografted mice and treated patients. The obvious implication is that to cure cancer all subpopulations should be the target and this leads us to ask: how many subpopulations actually exist in a given tumor and how different are they from each other? How many drugs do we need to target all of them?

This article will emphasize the general effect and outcomes we can expect from CSCs/CS-LCs targeting drugs and/or the tumor microenvironment according to our present knowledge of cancer biology. Providing an entire update of the list of CSCs/CS-LCs targeting drugs is beyond the scope of this review. However, reviews covering these specific topics have been recently published and are referred to through this article.
Correspondence: Juan Sebastian Yakisich, Department of Clinical Neuroscience, Karolinska University Hospital, Stockholm, Sweden, SE-14186. Tel. +46.8.585.89.533 - Fax: +46.8.585.83810. E-mail: sebastian.yakisich@ki.se

Key words: cancer, stem cells, proliferation, microenvironment, targeted therapy, stemness, signaling pathways, chemotherapy.

Conflict of interests: there is no conflict of interests with this research.

Funding: research in the author's lab is supported by grants from the Swedish Research Council and the Karolinska Institute.

Received for publication: 19 January 2012. Accepted for publication: 16 March 2012.

This work is licensed under a Creative Commons Attribution NonCommercial 3.0 License (CC BYNC 3.0).

(OCopyright J.S. Yakisich et al., 2012 Licensee PAGEPress, Italy

Drugs and Therapy Studies 2012; 2:e10

doi:10.4081/dts.2012.e10

\section{Cancer stem cells/cancer stem- like cells targeting drugs}

Selectively targeting CSCs/CS-LCs was initially considered a very promising strategy to cure cancer. In theory, any specific cancer stem cell marker is a potential target and attempts have been made to formulate strategies to exploit them. The limitations of the cancer stem cell markers will likely be translated into clinical failure when trying to target these markers. For instance, targeting $\mathrm{CD} 133^{+}$cells with monoclonal antibodies for glioma treatment will serve no purpose since it will spare the CD133- cells that can repopulate the original tumor. Monoclonal antibodies targeting other surface markers (e.g. CD123, CD44, CD33, and CD326) have been developed and are under evaluation. As expected, some of them, such as gemtuzumab ozogamicin (Mylotarg) and lintuzumab ${ }^{18}$ and bivatuzumab mertansine ${ }^{19}$ have, in the best cases, shown only modest benefit and have been discontinued. Despite the extensive list of potential specific CSCs/CS-LCs targeting drugs, for the moment, few drugs that selectively kill these cells have been identified (Table 1). ${ }^{20-31}$ Salinomycin was identified as selective inhibitors of cancer stem cells by high-throughput screening from a collection of 16,000 compounds. ${ }^{20}$ It was shown to induce apoptosis in human $\mathrm{CD} 4^{+}$T-cell 
leukemia cells, but not in normal $\mathrm{CD}^{+} \mathrm{T}$ cells. ${ }^{24}$ However, salinomycin is very toxic to other normal cells at concentrations effective against CSCs. ${ }^{32}$ Thus, it is unlikely that salinomycin will be useful as a single agent. ${ }^{33}$

The identification of signaling pathways important for maintaining the stemness phenotype was received with great enthusiasm and several small molecules are currently being investigated. The signaling pathways that can be targeted include Hedgehog, Notch and Wnt, among others. ${ }^{34,35}$ For instance, cyclopamine $^{36}$ and SANT-1 target the Hedgehog pathway that may contribute to the induction and maintenance of pancreatic ${ }^{37}$ and breast $^{38}$ tumors. During the period 1999-2009, at least 44 novel substances have been patented as inhibitors of the Hedgehog pathway ${ }^{39}$ and many others (including some activators) are being tested. ${ }^{40}$ Some novel compounds (GDC-0449 and IPI-926) have entered clinical trials. ${ }^{41}$ ZTM000990 and PKF118-310 target the canonical WNT signaling cascade ${ }^{35}$ and gamma-secretase inhibitor GSI-18 is active against the Notch signaling pathway. ${ }^{6,42}$ Most of these substances are in the very early phase of pre-clinical testing. Similar to salinomycin, they may be of limited use as single agents. Other available substances, while not selective CSCs/CS-LCs inhibitors or selective targeting stemness signaling pathways, are able to modify the stemness properties of cancer cells (Table 2). ${ }^{43-57}$
Therefore, at least three classes of stemness modulating drugs (SMDs) have been identified.

i) Stem cell targeting drugs (SCTDs) selectively kill CSC/CS-LCs sparing non-CSCs. Salinomycin may be a prototype of this class but it use may be limited by its high toxicity. ${ }^{33}$ Other SCTDs have been reported (Table 1).

ii) Stemness inhibiting drugs (SIDs) do not kill CSCs/CS-LCs but reduce the stemness properties of the cancer cells. Few drugs with these properties have been identified (Table 2). Although there is little distinction between an SCTD and an SID (SID at high doses may eliminate CSCs/CS-LCs), they are conceptually different classes of drugs. While an SCTD eliminates CSCs/CS-LCs at concentrations that are much less toxic to non-CSCs (e.g. salinomycin, see below), an SID may eliminate non-CSCs and CSCs/CS-LCs with similar potency but at the same time reduce the stemnnes of CSCs/CS-LCs.

iii) Stemness promoting drugs (SPDs) do not kill CSCs/CS-LCs but increase the stemness properties of the cancer cells. So far, to our knowledge, only metformin has been reported to have this property (Table 2).

Table 1. Examples of selective cancer stem cells targeting drugs (SCTDs).

\begin{tabular}{llc}
\hline Agent & Cancer type & Refs. \\
Salinomycin & Breast & 20 \\
& Gastric & 21 \\
& Prostate & 22 \\
& Osteosarcoma & 23 \\
& Acute T-cell leukemia & 24 \\
& Chronic lymphocytic leukemia & 25 \\
& Human promyeloblastic leukemia & 26 \\
& Colorectal & 27 \\
$3-0-m e t h y l f u n i c o n e$ & Lung adenocarcinoma & 28 \\
LSD1 inhibitors & Breast & 29 \\
Cyclopamine & Several pluripotent germ cell tumors (F9, NCCIT, NTERA-2) & 30 \\
\hline
\end{tabular}

Table 2. Examples of stemness modulator drugs (SMDs).

\begin{tabular}{|c|c|c|c|}
\hline Cell line/cancer & Agent & $\begin{array}{l}\text { Effect on stemness / } \\
\text { (effect on cytotoxicity) }\end{array}$ & Refs. \\
\hline U87MG and U373MG (glioma) & Eckol & $\downarrow /(\uparrow)$ & 43 \\
\hline U87 (glioma) & Nordy & $\downarrow /$ (N.A.) & 44 \\
\hline HCT116 (colorectal) & Lovastatin & $\downarrow /(\uparrow)$ & 45 \\
\hline SW1990 (gemcitabine-resistant pancreatic cell line) & Cyclopamine & $\downarrow(\uparrow)$ & 46 \\
\hline Breast & SANT-1 & $\downarrow(\uparrow)$ & 38 \\
\hline Pancreas & SANT-1 & $\downarrow(\uparrow)$ & 37 \\
\hline Patient-derived (glioma) & Resveratrol & $\downarrow /(\uparrow)$ & 47 \\
\hline Patient-derived (glioma) & AG490 & $\downarrow /(\uparrow)$ & 47 \\
\hline Patient NSCLC-derived cells (lung) & Cucurbitacin & $\downarrow /(\uparrow)$ & 48 \\
\hline Patient HNSCC-derived cells (Head and neck squamous cell carcinoma) & Cucurbitacin & $\downarrow /(\uparrow)$ & 49 \\
\hline MCF-7 (breast) & Metformin & $\uparrow /($ N.A.) & 50 \\
\hline MCF-7 (breast) & Berberine & $\downarrow /$ (N.A.) & 51 \\
\hline FOLFOX-resistant cells (colon cancer) & Curcumin & $\downarrow /(\uparrow)$ & 52 \\
\hline Glioma & ER-400583-00 & $\downarrow(\uparrow)$ & 53 \\
\hline Glioma & WP1193 & $\downarrow(\uparrow)$ & 54 \\
\hline Glioma & Angiogenesis inhibitors & $\downarrow(\uparrow)$ & 55 \\
\hline Glioma & All-trans retinoic acid & $\downarrow(\uparrow)$ & 56 \\
\hline \multicolumn{4}{|l|}{ Non-cancer } \\
\hline 3T3-L1 preadipocytes & Curcumin & $\uparrow /$ (N.A.) & 57 \\
\hline
\end{tabular}




\section{Combination therapy using stemness modulator drugs}

Combination therapy using monoclonal antibodies against stem cell markers and standard anticancer drugs did not show any great benefit. Recent Phase II studies of combination therapy with gemtuzumab ozogamicin (G0), e.g. $\mathbf{G 0}+$ vorinostat, ${ }^{58} \mathrm{GO}+$ ara- $\mathrm{C},{ }^{59} \mathrm{GO}$ + arsenic trioxide ${ }^{60}$ and GO + fludarabine, cytarabine, idarubicin (FLAI-G0), ${ }^{61}$ showed limited efficacy or, in the best cases, encouraging results that need further evaluation in multicenter trials.

Several combinations of an SMD + standard anticancer drug (SAD) have reported (Table $3)^{62-72}$ potential advantages including a more potent anticancer effect with lower toxicity to normal cells. However, most of those studies did not evaluate the ability of the combination agents to actually deplete all cancer cells when that should be the ultimate goal. This is in part because most of the in vitro assays to test these drug combinations use short term assays $(24-72 \mathrm{~h})$ that are not adequate to evaluate the fate of surviving cells. ${ }^{73}$ It is possible that the encouraging effect of these combinations (SMD+SAD) will be no better than any other standard combination (SAD1+SAD2) and that after treatment they will leave a fraction of resistant surviving cells that will lead to a tumor relapse. For a successful treatment (i.e. a cure) these cells need to be eliminated.

\section{Targeting the microenviron- ment and lessons from the anti-angiogenesis hypothesis}

The microenvironment plays an important role in determining the stemness properties of cancer cells and may constitute an important target. ${ }^{74-76}$ Microenvironment targeting drugs (MMDs) are also being developed (Table 4) (7-85 $^{7}$ but one can anticipate the same type of problems and ask the same questions as before for targeting CSCs/CS-LCs: how many microenvironments actually exist in a given tumor and how different are they from each other? How many drugs do we need to target all of them? In fact, cancer researchers have been targeting the microenvironment without success for almost 40 years with angiogenesis inhibitors Angiogenesis is the formation of new blood vessels and since tumors need blood vessels to grow and spread it was postulated that blocking this process would stop cancer growth. The idea was received with great enthusiasm. Up till now, hundreds of angiogenesis inhibitors have been developed and tested but only a few have shown any benefit in clinical trials and none of them succeed in curing cancer. It seems that angiogenesis inhibition is not enough $^{86}$ either alone or in combination with other anticancer modalities. For advanced melanomas, around 70 angiogenesis inhibitors with different mechanisms of actions are in clinical experimental use but this disease still has a poor prognosis with an average survival of less than one year. ${ }^{84}$ To date, angiogenesis inhibition has only improved progres-

Table 3. Examples of combination therapy with stemness modulatordrug and standard anticancer drugs.

\begin{tabular}{|c|c|c|c|}
\hline $\begin{array}{l}\text { Combination } \\
\text { Stemness modulatordrug (SMD) }\end{array}$ & Standard anticancer drug (SAD) & Cancer type & Refs. \\
\hline Salinomycin & Gemcitabine & Pancreatic & 62 \\
\hline Salinomycin & Octreotide modified Paclitaxel & Breast & 63 \\
\hline Salinomycin & $\begin{array}{l}\text { Etoposide } \\
\text { Doxorubicin }\end{array}$ & $\begin{array}{l}\text { Hepatic } \\
\text { Uterine sarcoma Breast }\end{array}$ & 64 \\
\hline SANT-1 & SAHA $>$ & Pancreatic & 65 \\
\hline GSI-XII & Bortezomib & Multiple Myeloma & 66 \\
\hline GSI-XII & ABT-737 & Multiple myeloma & 67 \\
\hline Curcumin & Dasatinib & Colon & $52,68,69$ \\
\hline Curcumin & Gemcitabine & Pancreatic & 70 \\
\hline Curcumin & $\begin{array}{l}\text { Gemcitabine } \\
\text { Placlitaxel } \\
\text { Tumor necrosis factor (TNF) } \\
\text { TNF-related apoptosis inducing ligand }\end{array}$ & Bladder & 71 \\
\hline Curcumin & 5-FU plus oxaliplatin (FOLFOX) & Colon & 72 \\
\hline ER-400583-00 & Radiation & Gliomas & 53 \\
\hline VEGFR2 targeting antibody & Cyclophosphamide & Gliomas & 55 \\
\hline
\end{tabular}

Table 4. Examples of microenvironment modulator drugs (MMDs).

\begin{tabular}{lc} 
Cell line/cancer & Agent \\
Pancreas & PF-562,271 \\
Different models of cancer & Monoclonal antibody (AB0023) \\
\hline Lung & Pazopanib \\
Human colon and gastric cancer xenograft & TSU68 \\
\hline Head and neck squamous cell carcinoma Non-small cell lung cancer & Erlotinib \\
Glioblastoma & QLT0254 \\
\hline Glioblastoma & QLT0267 \\
Melanoma & 80,81 \\
\hline Gliomas & Angiogenesis inhibitors \\
\hline
\end{tabular}


sion-free survival in highly vascular tumors such as gliomas, ${ }^{85,87,88}$ ovarian cancer $^{89}$ or hepatocellular carcinoma, ${ }^{90}$ but no cure has been achieved. The response of the cancer community to the cancer stem cell hypothesis has been in some ways similar to the idea introduced around 40 years ago to treat cancer by inhibiting angiogenesis. The pharmacological search for the elimination or modulation of cancer stem cells seems to sum up the angiogenesis story: the pioneer identification of putative stem cells in human acute myeloid leukemia in 1997, ${ }^{91}$ and later in 2003 in breast cancer ${ }^{92}$ and brain tumors, ${ }^{5}$ gave rise to extensive research on the biology of CSCs/CS-LCs as well as on the development of pharmacological agents targeting these cells. Equally disappointing, after almost 15 years of cancer stem cell research, anticancer drugs targeting CSCs/CS-LCs have shown only modest clinical benefit.

Substances that inhibit or indirectly modulate the expression of the hypoxia-inducible factor-1 (HIF-1), such a konokiol, ${ }^{93}$ manassantin B and 4-0-demethylmanassantin B, ${ }^{94}$ laurenditerpenol, ${ }^{95}$ curcumin, ${ }^{96}$ trans-3, $4,5^{\prime}$-trihydroxystibene, ${ }^{97}$ resveratrol, ${ }^{98}$ SU5416, ${ }^{99}$ are also modulators of the microenvironment. However, they showed little efficacy either alone or in combination with standard anticancer agents or radiation. Around $30 \mathrm{HIF}$ inhibitors are currently being investigated in clinical trials ${ }^{90}$ but it has already been shown that some of them fail to cure cancer.

The finding that a subset of glioblastoma stem-like cells show characteristics of endothelial progenitors and are capable of maturation into endothelial cells, ${ }^{100}$ if confirmed for other types of tumors, further supports the idea that all cancer cells should be eliminated at once and that specific subpopulations of cancer cells or specific microenvironments per se are minor targets in the complex tumoral tissue.

\section{Anticipated problems for tar- geting cancer stem cells/cancer stem-like cells or the tumour microenvironment}

The heterogeneous nature of CSCs/CS-LCs may represent an important problem for the selective targeting of specific cancer cell subpopulations, stemness-related signaling pathways or specific tumor microenvironments. For instance, metastatic colon cancer HCT116 cells express the majority of known CSC markers but also show markedly phenotypic variation indicating that CSCs represent a heterogeneous population. ${ }^{101}$ These signaling path- ways per se are not valuable targets for anticancer therapy. It is likely that, due to the high intratumoral heterogeneity, the functional status of a particular signaling pathway can be highly active in one cell phenotype, relatively inactive in another and close to normal in a third phenotype within the same tumor. A small molecule targeting this particular pathway will only be effective on the cells that belong to the first group. As discussed above, a similar outcome can be expected with MMDs.

\section{A modular tumor model}

In gliomas, radioresistance seems to be the result of interaction between cancer cells and the microenvironment that creates a microenvironment-stem cell unit and there is extensive data to suggest that $\mathrm{CSCs} / \mathrm{CS}$-LCs reside in specific niches (microenvironment). ${ }^{102}$ If this is true, it is obvious that there are other specific niches where non-CSCs and other cancer cell subpopulations reside. This suggests that complex, highly heterogeneous tumors may be organized in modules of specific cancer cell phenotype in their specific microenvironment (modular tumor model (MTM)). Each module is a microenvironmentcancer cell unit enriched for cells with a specific stemness phenotype. The interaction between the microenvironment and their associated cells determines the chemosensitivity of the entire module for a particular anticancer agent. Figure 1 shows the schematic represen-

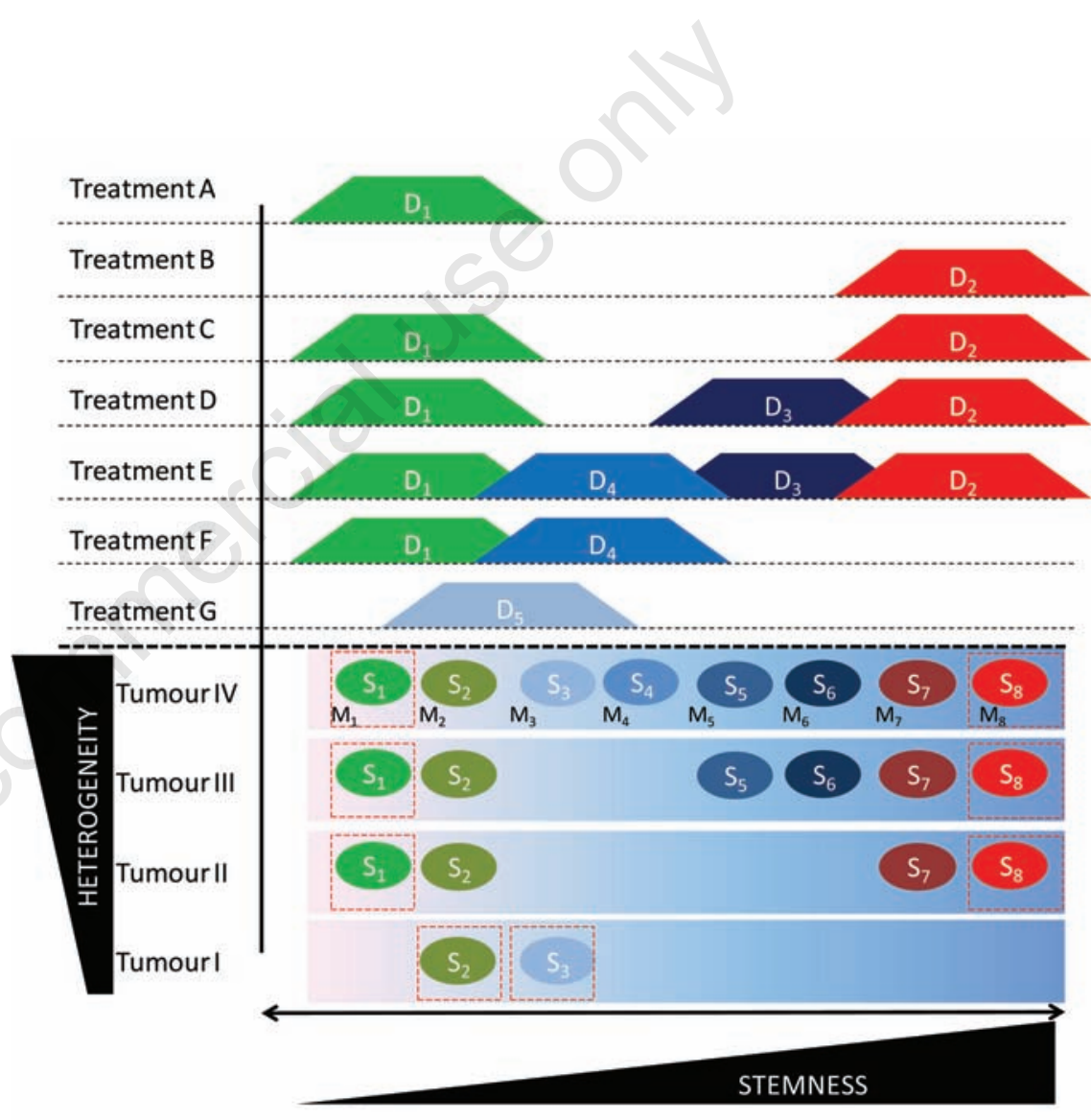

Figure 1. The modular tumor model (MTM). S1-S8 represent cancer cells with different stemness phenotypes (subpopulations) in their respective microenvironment (M1-M8) that creates specific microenvironment-cancer cell units (e.g. M1/S1or M8/S8). D1-D5 represents drugs or a combination of drugs that preferentially eliminate a specific cell phenotype or target a specific microenvironment (that in turn eliminates a subpopulation of cancer cells). Their position in the chart indicates the target (e.g. D1 targets S1$\mathrm{S} 2$ and/or M1-M2). Treatments A-G represent the use of D1-D5 as single agents or in combination and the predicted outcome depending on the tumor heterogeneity. A highly homogeneous tumor (Tumor I) will be easily cured with one (D5, treatment $G$ ) or two drugs (D1 and/or D4, treatment F). As tumor heterogeneity increases (from Tumor I to Tumor IV), the number of drugs necessary to eradicate the tumor also increases. Treatments A and B (single agents) will be ineffective. Treatment $D$ will be effective for a Tumor I, II and III but ineffective for Tumor IV since it will spare the M3/S3 and M4/S4 modules. For simplicity, only 8 different continuous modules (showed as a gradient from $\mathrm{M} 1 / \mathrm{S} 1$ to $\mathrm{M8/S8)}$ have been presented in this model. 
tation of tumors of increasing heterogeneity and an increasing number of microenvironment-cancer cell units. Due to the plastic nature of cancer cells, changes in the microenvironment (e.g. drugs) (Table 2) can eliminate or affect the stemness of the associated cells. Highly homogeneous tumors are organized in only a few modules and highly heterogeneous tumors in many. The increasing complexity dictates the number of drugs necessary to eliminate all modules. It is likely that the most curable cancers (e.g. testicular cancer ${ }^{103}$ or acute promyelocytic leukemia (APL) ${ }^{104}$ are the most simple and are organized in just a few microenvironment-cancer cell modules (similar to Tumor I in Figure 1). Indeed, APL, a rare but highly homogeneous cancer, can be cured in most cases with a combination of all-trans retinoic acid (ATRA) and arsenic trioxide (AT0). ${ }^{104,105}$ These drugs also target the leukemia-initiating cells. ${ }^{106,107}$ For highly heterogeneous tumors, usually associated with a poor prognosis, it will be necessary to identify and characterize all relevant modules in order to develop effective cancer therapies.

\section{Conclusions}

The complex cellular heterogeneity of cancers and the growing evidence that tumors contain cells with different stem cell phenotypes, that in turn reside in specific microenvironment rather than a pure CSC and a nonCSC subpopulation, challenges and limits the clinical use of SMDs and/or MMDs. Despite the enormous interest and apparent strong rationale to develop SMDs and/or MMDs, the chances of obtaining a cure are slim since we do not know how many subpopulations (or phenotypes) of cancer cells and specific microenvironments need to be targeted. At present, there is still a translational gap between the basic research and pre-clinical anticancer drug screening. On a basic level, we are aware of the complexity of tumors and recognize the need for therapies aimed at eliminating all cancer cells. But most in vitro pre-clinical studies and tests in animal models are usually designed to target only a few cancer cell phenotypes. This translates into poor clinical outcome since most clinical trials are also designed for testing single agents or combinations of just a few (usually 2-3) drugs that will likely eliminate only a few cancer cell subpopulations. Unless we develop a more integrated approach to eliminate all cancer cells at once rather than target a specific pathway, cell subtype or microenvironment, the next 25 years will only serve to repeat the experience of 40 years of angiogenesis research. We will get a better understanding of cancer stem cell biology and tumor microenvironment, and produce and test hundreds or thousands of novel drugs, but very limited clinical success will be achieved. The real challenge is to find a way to integrate advances in cancer biology into successful therapies that save lives. The MTM may serve as a useful guide to develop cancer treatment regimes in a more rational way, based on our knowledge of the complexity of tumor biology in terms of cancer stem cells and their interaction with the tumor microenvironment.

\section{References}

1. Albini A, Cesana E, Noonan DM. Cancer stem cells and the tumor microenvironment: soloists or choral singers. Curr Pharm Biotechnol 2011;12:171-81.

2. Brennan SK, Matsui W. Cancer stem cells: controversies in multiple myeloma. J Mol Med 2009;87:1079-85.

3. Cruz M, Siden $\AA$, Tasat DR,Yakisich JS. Are all glioma cells cancer stem cells? J Cancer Sci Ther 2010;2:100-6.

4. Rowan K. Are Cancer Stem Cells Real? After Four Decades, Debate Still Simmers. JNCI 2009;101:546.

5. Singh SK, Clarke ID, Terasaki M, et al. Identification of a cancer stem cell in human brain tumors. Cancer Res 2003;63: 5821-8.

6. Fan X, Matsui W, Khaki L, et al. Notch pathway inhibition depletes stem-like cells and blocks engraftment in embryonal brain tumors. Cancer Res 2006;66: 7445-52.

7. Liu G, Yuan X, Zeng Z, et al. Analysis of gene expression and chemoresistance of CD133+ cancer stem cells in glioblastoma. Mol Cancer 2006;5:67.

8. Beier D, Röhrl S, Pillai DR, et al. Temozolomide preferentially depletes cancer stem cells in glioblastoma. Cancer Res 2008;68:5706-6715.

9. Wang J, Sakariassen PØ, Tsinkalovsky 0, et al. CD133 negative glioma cells form tumors in nude rats and give rise to CD133 positive cells. Int $\mathrm{J}$ Cancer 2008;122:761-8.

10. Zheng X, Shen G, Yang X, Liu W. Most C6 cells are cancer stem cells: evidence from clonal and population analyses. Cancer Res. 2007;67:3691-7. Erratum in: Cancer Res 2007;67:10097.

11. Bidlingmaier S, Zhu X, Liu B. The utility and limitations of glycosylated human CD133 epitopes in defining cancer stem cells. J Mol Med 2008;86:1025-32.
12. Meng $\mathrm{X}$, Li M, Wang $\mathrm{X}$, et al. Both CD133+ and CD133- subpopulations of A549 and H446 cells contain cancer-initiating cells. Cancer Sci 2009;100:1040-6.

13. Castriconi R, Dondero A, Negri F, et al. Both CD133+ and CD133- medulloblastoma cell lines express ligands for triggering NK receptors and are susceptible to NK-mediated cytotoxicity. Eur J Immunol 2007;37:3190-6.

14. Bao S, Wu Q, McLendon RE, et al. Glioma stem cells promote radioresistance by preferential activation of the DNA damage response. Nature 2006;444:756-60.

15 Ropolo M, Daga A, Griffero F, et al. Comparative analysis of DNA repair in stem and nonstem glioma cell cultures. Mol Cancer Res 2009;7:383-92.

16. Liu T, Xu F, Du X, et al. Establishment and characterization of multi-drug resistant, prostate carcinoma-initiating stem-like cells from human prostate cancer cell lines 22RV1. Mol Cell Biochem 2010;340:265-73.

17. Ma L, Lai D, Liu T, et al. Cancer stem-like cells can be isolated with drug selection in human ovarian cancer cell line SKOV3. Acta Biochim Biophys Sin 2010;42:593602 .

18. Jurcic JG. What Happened to Anti-CD33 Therapy for Acute Myeloid Leukemia? Curr Hematol Malig Rep 2011;7:65-73.

19. Scatena R, Bottoni P, Pontoglio A, Giardina B. Cancer stem cells: the development of new cancer therapeutics. Expert Opin Biol Ther. 2011;7:875-92.

20. Gupta PB, Onder TT, Jiang G, et al. Identification of selective inhibitors of cancer stem cells by high-throughput screening. Cell 2009;138:645-59.

21. Zhi QM, Chen XH, Ji J, et al. Salinomycin can effectively kill ALDH(high) stem-like cells on gastric cancer. Biomed Pharmacother 2011;65:509-15.

22. Kim KY, Yu SN, Lee SY, et al. Salinomycin-induced apoptosis of human prostate cancer cells due to accumulated reactive oxygen species and mitochondrial membrane depolarization. Biochem Biophys Res Commun 2011;413:80-6.

23. Tang QL, Zhao ZQ, Li JC, et al. Salinomycin inhibits osteosarcoma by targeting its tumor stem cells. Cancer Lett 2011;311:113-21.

24. Fuchs D, Heinold A, Opelz G, et al. Salinomycin induces apoptosis and overcomes apoptosis resistance in human cancer cells. Biochem Biophys Res Commun 2009;390:743-9.

25. Lu D, Choi MY, Yu J, et al. Salinomycin inhibits Wnt signaling and selectively induces apoptosis in chronic lymphocytic 
leukemia cells. Proc Natl Acad Sci USA 2011;108:13253-7.

26. Fuchs D, Daniel V, Sadeghi M, et al. Salinomycin overcomes ABC transportermediated multidrug and apoptosis resistance in human leukemia stem cell-like KG-1a cells. Biochem Biophys Res Commun 2010;394:1098-104.

27. Dong TT, Zhou HM, Wang LL, et al. Salinomycin selectively targets 'CD133+' cell subpopulations and decreases malignant traits in colorectal cancer lines. Ann Surg Oncol 2011;18:1797-804.

28. Wang Y. Effects of salinomycin on cancer stem cell in human lung adenocarcinoma A549 cells. Med Chem 2011;7:106-11.

29. Buommino E, Tirino V, De Filippis A, et al. 3-0-methylfunicone, from Penicillium pinophilum, is a selective inhibitor of breast cancer stem cells. Cell Prolif 2011; 44:401-9.

30. Wang J, Lu F, Ren Q, et al. Novel Histone Demethylase LSD1 Inhibitors Selectively Target Cancer Cells with Pluripotent Stem Cell Properties. Cancer Res 2011;71:7238-49.

31. Bar EE, Chaudhry A, Lin A, et al. Cyclopamine-mediated hedgehog pathway inhibition depletes stem-like cancer cells in glioblastoma. Stem Cells 2007;25:2524-33.

32. Boehmerle W, Endres M. Salinomycin induces calpain and cytochrome c-mediated neuronal cell death. Cell Death Dis 2011;2:e168.

33. Delwar ZM, Avramidis D, Siden Å, et al. Depletion of drug-surviving glioma cells by a second phase treatment with low concentration of salinomycin. 2011;1:215 .

34. Bates SE. Drugs that target the stemness pathways. Clin Cancer Res 2010;16:3105.

35. Katoh M, Katoh M. WNT signaling pathway and stem cell signaling network. Clin Cancer Res 2007;13:4042-5.

36. Gould A, Missailidis S. Targeting the hedgehog pathway: the development of cyclopamine and the development of anti-cancer drugs targeting the hedgehog pathway. Mini Rev Med Chem 2011; 11:200-13.

37. Xu FG, Ma QY, Wang Z. Blockade of hedgehog signaling pathway as a therapeutic strategy for pancreatic cancer. Cancer Lett 2009;283:119-24.

38. Tanaka H, Nakamura M, Kameda C, et al. The Hedgehog signaling pathway plays an essential role in maintaining the CD44+CD24-/low subpopulation and the side population of breast cancer cells. Anticancer Res 2009;29:2147-57.

39. Tremblay MR, Nesler M, Weatherhead R, Castro AC. Recent patents for Hedgehog pathway inhibitors for the treatment of malignancy. Expert Opin Ther Pat 2009;19:1039-56.

40. Heretsch P, Tzagkaroulaki L,Giannis A. Modulators of the hedgehog signaling pathway. Bioorg Med Chem. 2010;18: 6613-24.

41. Stanton BZ, Peng LF. Small-molecule modulators of the Sonic Hedgehog signaling pathway. Mol Biosyst 2010;6:4454.

42. Mullendore ME, Koorstra JB, Li YM, et al. Ligand-dependent Notch signaling is involved in tumor initiation and tumor maintenance in pancreatic cancer. Clin Cancer Res 2009;15:2291-3201.

43. Hyun KH, Yoon CH, Kim RK, et al. Eckol suppresses maintenance of stemness and malignancies in glioma stem-like cells. Toxicol Appl Pharmacol 2011;254: $32-40$.

44. of arachidonate 5-lipoxygenase, Nordy, induces differentiation and inhibits selfrenewal of glioma stem-like cells. Stem Cell Rev 2011;7:458-70.

45. Kodach LL, Jacobs RJ, Voorneveld PW, et al. Statins augment the chemosensitivity of colorectal cancer cells inducing epigenetic reprogramming and reducing colorectal cancer cell 'stemness' via the bone morphogenetic protein pathway. Gut 2011;60:1544-53.

46. Yao J, An Y, Wie JS, et al. Cyclopamine reverts acquired chemoresistance and down-regulates cancer stem cell markers in pancreatic cancer cell lines. Swiss Med Wkly 2011;141:w13208.

47. Yang YP, Chang YL, Huang PI, et al. Resveratrol suppresses tumorigenicity and enhances radiosensitivity in primary glioblastoma tumor initiating cells by inhibiting the STAT3 axis. J Cell Physiol 2011;227:976-93.

48. Hsu HS, Huang PI, Chang YL, et al. Cucurbitacin i inhibits tumorigenic ability and enhances radiochemosensitivity in nonsmall cell lung cancer-derived CD133-positive cells. Cancer 2011;117:2970-85.

49. Chen YW, Chen KH, Huang PI, et al. Cucurbitacin I suppressed stem-like property and enhanced radiation-induced apoptosis in head and neck squamous carcinoma--derived CD44(+)ALDH1(+) cells. Mol Cancer Ther 2010;9:2879-92.

50. Oliveras-Ferraros C, Vazquez-Martin A, Menendez JA. Pharmacological mimicking of caloric restriction elicits epigenetic reprogramming of differentiated cells to stem-like self-renewal states. Rejuvenation Res 2010;13:519-26.

51. Kim JB, Ko E, Han W, et al. Berberine diminishes the side population and ABCG2 transporter expression in MCF-7 breast cancer cells. Planta Med
2008;74:1693-700.

52. Nautiyal J, Kanwar SS, Yu Y, Majumdar AP. Combination of dasatinib and curcumin eliminates chemo-resistant colon cancer cells. J Mol Signal 2011;6:7.

53. Okamoto K, Ito D, Miyazaki $\mathrm{K}$, et al. Microregional antitumor activity of a small-molecule hypoxia-inducible factor 1 inhibitor. Int J Mol Med 2012;29:541-9.

54. Sai K, Wang S, Balasubramaniyan V, et al. Induction of cell-cycle arrest and apoptosis in glioblastoma stem-like cells by WP1193, a novel small molecule inhibitor of the JAK2/STAT3 pathway. J Neurooncol 2012;107:487-501.

55. Folkins C, Man S, Xu P, et al. Anticancer therapies combining antiangiogenic and tumor cell cytotoxic effects reduce the tumor stem-like cell fraction in glioma xenograft tumors. Cancer Res 2007;67:3560-4.

56. Campos B, Wan F, Farhadi M, et al. Differentiation therapy exerts antitumor effects on stem-like glioma cells. Clin. Cancer Res 2010;16:2715-28.

57. Kim JH, Park SH, Nam SW, et al. Curcumin stimulates proliferation, stemness acting signals and migration of 3T3L1 preadipocytes. Int J Mol Med 2011;28: 429-35.

58. Walter RB, Medeiros BC, Powell BL, et al. Phase 2 trial of vorinostat and gemtuzumab ozogamicin as induction and post-remission therapy in older adults with previously untreated acute myeloid leukemia. Haematologica 2011;97:73942.

59. Brunnberg U, Mohr M, Noppeney R, et al. Induction therapy of AML with ara-C plus daunorubicin versus ara-C plus gemtuzumab ozogamicin: a randomized phase II trial in elderly patients. Ann Oncol 2011;23:990-6.

60. Sekeres MA, Maciejewski JP, Erba HP, et al. A Phase 2 study of combination therapy with arsenic trioxide and gemtuzumab ozogamicin in patients with myelodysplastic syndromes or secondary acute myeloid leukemia. Cancer 2011;117: 1253-61.

61. Candoni A, Martinelli G, Toffoletti E, et al. Gemtuzumab-ozogamicin in combination with fludarabine, cytarabine, idarubicin (FLAI-G0) as induction therapy in CD33positive AML patients younger than 65 years. Leuk Res 2008;32:1800-8.

62. Combination of salinomycin and gemcitabine eliminates pancreatic cancer cells. Cancer Lett 2011;313:137-44.

63. Zhang Y, Zhang H, Wang X, et al. The eradication of breast cancer and cancer stem cells using octreotide modified paclitaxel active targeting micelles and salinomycin passive targeting micelles. 
Biomaterials 2012;33:679-91.

64. Kim JH, Chae M, Kim WK, et al. Salinomycin sensitizes cancer cells to the effects of doxorubicin and etoposide treatment by increasing DNA damage and reducing p21 protein. Br J Pharmacol 2011;162:773-84.

65. Chun SG, Zhou W, Yee NS. Combined targeting of histone deacetylases and hedgehog signaling enhances cytoxicity in pancreatic cancer. Cancer Biol Ther 2009;8:1328-3139.

66. Chen F, Pisklakova A, Li M, et al. Gammasecretase inhibitor enhances the cytotoxic effect of bortezomib in multiple myeloma. Cell Oncol 2011;34:545-51.

67. Li M, Chen F, Clifton N, et al. Combined inhibition of Notch signaling and Bcl2/Bcl-xL results in synergistic antimyeloma effect. Mol Cancer Ther 2010;9:32009 .

68. Nautiyal J, Banerjee S, Kanwar SS, et al. Curcumin enhances dasatinib-induced inhibition of growth and transformation of colon cancer cells. Int J Cancer 2011 128:951-61.

69. Patel BB, Sengupta R, Qazi S, et al. Curcumin enhances the effects of 5-fluorouracil and oxaliplatin in mediating growth inhibition of colon cancer cells by modulating EGFR and IGF-1R. Int J Cancer 2008;122:267-73.

70. Kunnumakkara AB, Guha S, Krishnan S, et al. Curcumin potentiates antitumor activity of gemcitabine in an orthotopic model of pancreatic cancer through suppression of proliferation, angiogenesis, and inhibition of nuclear factor-kappaBregulated gene products. Cancer Res 2007;67:3853-61.

71. Kamat AM, Sethi G, Aggarwal BB. Curcumin potentiates the apoptotic effects of chemotherapeutic agents and cytokines through down-regulation of nuclear factor-kappaB and nuclear factorkappa B-regulated gene products in IFNalpha-sensitive and IFN-alpha-resistant human bladder cancer cells. Mol Cancer Ther 2007;6:1022-30.

72. Yu Y, Kanwar SS, Patel BB, et al. Elimination of Colon Cancer Stem-Like Cells by the Combination of Curcumin and FOLFOX. Transl Oncol 2009;2:321-8.

73. Avramidis D, Cruz M, Sidén A, et al. Regrowth Concentration Zero (RC0) as complementary endpoint parameter to evaluate compound candidates during preclinical drug development for cancer treatment. J. Cancer Sci Ther 2009;1:1924.

74. Guise T. Examining the metastatic niche: targeting the microenvironment. Semin Oncol 2010;37:S2-14.

75. Karar J, Maity A. Modulating the tumor microenvironment to increase radiation responsiveness. Cancer Biol Ther 2009;8: 1994-2001.

76. Zocchi MR, Poggi A. Targeting the Microenvironment in Hematological Malignancies: How to Condition both Stromal and Effector Cells to Overcome Cancer Spreading. Curr Med Chem 2011; 18: 5172-3.

77 Stokes JB, Adair SJ, Slack-Davis JK, et al. Inhibition of focal adhesion kinase by PF562,271 inhibits the growth and metastasis of pancreatic cancer concomitant with altering the tumor microenvironment. 2011;10:2135-45.

78. Barry-Hamilton V, Spangler R, Marshall $\mathrm{D}$, et al. Allosteric inhibition of lysyl oxidase-like-2 impedes the development of a pathologic microenvironment. Nat Med 2010;16:1009-17.

79. Tailor TD, Hanna G, Yarmolenko PS, et al. Effect of pazopanib on tumor microenvironment and liposome delivery. Mol Cancer Ther 2010;9:1798-808.

80. Ohta M, Kawabata T, Yamamoto M, et al. TSU68, an antiangiogenic receptor tyrosine kinase inhibitor, induces tumor vascular normalization in a human cancer xenograft nude mouse model. Surg Today 2009;39:1046-53.

81. Yamamoto M, Kikuchi H, Ohta M, et al. TSU68 prevents liver metastasis of colon cancer xenografts by modulating the premetastatic niche. Cancer Res 2008;68:9754-62.

82. Cerniglia GJ, Pore $\mathrm{N}$, Tsai JH, et al. Epidermal growth factor receptor inhibition modulates the microenvironment by vascular normalization to improve chemotherapy and radiotherapy efficacy. PLoS One 2009;4:e6539.

83. Edwards LA, Woo J, Huxham LA, et al. Suppression of VEGF secretion and changes in glioblastoma multiforme microenvironment by inhibition of integrin-linked kinase (ILK). Mol Cancer Ther 2008;7:59-70.

84. Bafaloukos DJ, Linardou H. Targeting angiogenesis for the treatment of advanced melanoma. Oncol Rev 2011;5:167-76

85. Takano S. Glioblastoma angiogenesis: VEGF resistance solutions and new strategies based on molecular mechanisms of tumor vessel formation. Brain Tumor Pathol 2012;29:73-86.

86. Roodink I, Leenders WP. Targeted therapies of cancer: angiogenesis inhibition seems not enough. Cancer Lett 2010; 299:1-10.

87. Brastianos PK, Batchelor TT. Vascular endothelial growth factor inhibitors in malignant gliomas. Target Oncol 2010;5: 167-74.
88. Morris PG. Bevacizumab is an active agent for recurrent high-grade glioma, but do we need randomized controlled trials? Anticancer Drugs 2012;in press.

89. Burger RA. Antiangiogenic agents should be integrated into the standard treatment for patients with ovarian cancer. Ann Oncol 2011;22:viii65-8.

90. Wenger JB, Santos N, Liu Y, et al. Can we develop effective combination antiangiogenic therapy for patients with hepatocellular carcinoma? Oncol Rev 2011;5: 177-84.

91. Bonnet D, Dick JE. Human acute myeloid leukemia is organized as a hierarchy that originates from a primitive hematopoietic cell. Nat Med 1997;3:730-7.

92. Al-Hajj M, Wicha MS, Benito-Hernandez A, et al. Prospective identification of tumorigenic breast cancer cells. Proc Natl Acad Sci USA 2003;100:6890.

93. Lan KL, Lan KH, Sheu ML, et al. Honokiol inhibits hypoxia-inducible factor-1 pathway. Int J Radiat Biol 2011;87:579-90.

94. Hodges TW, Hossain CF, Kim YP, et al Molecular-targeted antitumor agents: the Saururus cernuus dineolignans manassantin B and 4-0-demethylmanassantin $B$ are potent inhibitors of hypoxia-activated HIF-1. J Nat Prod 2004;67:767-71.

95. Mohammed KA, Hossain CF, Zhang L, et al. Laurenditerpenol, a new diterpene from the tropical marine alga Laurenciaintricata that potently inhibits HIF-1 mediated hypoxic signaling in breast tumor cells. J Nat Prod 2004;67:2002-7.

96. Choi H, Chun YS, Kim SW, et al. Curcumin inhibits hypoxia-inducible factor-1 by degrading aryl hydrocarbon receptor nuclear translocator: a mechanism of tumor growth inhibition. Mol Pharmacol 2006;70:1664-71.

97. Cao Z, Fang J, Xia C, et al. trans-3,4,5'Trihydroxystibene inhibits hypoxiainducible factor lalpha and vascular endothelial growth factor expression in human ovarian cancer cells. Clin Cancer Res 2004;10:5253-63.

98. Zhang $Q$, Tang X, Lu QY, et al. Resveratrol inhibits hypoxia-induced accumulation of hypoxia-inducible factor-lalpha and VEGF expression in human tongue squamous cell carcinoma and hepatoma cells. Mol Cancer Ther 2005;4:1465-74.

99. Zhong XS, Zheng JZ, Reed E, Jiang BH SU5416 inhibited VEGF and HIF-1alpha expression through the PI3K/AKT/p70S6K1 signaling pathway. Biochem Biophys Res Commun 2004;324: 471-80.

100. Wang R, Chadalavada K, Wilshire J, et al. Glioblastoma stem-like cells give rise to tumour endothelium. Nature 2010;468: 829-33. 
101. Botchkina IL, Rowehl RA, Rivadeneira DE, et al. Phenotypic subpopulations of metastatic colon cancer stem cells: genomic analysis. Cancer Genomics Proteomics 2009;6:19-29.

102. Mannino M, Chalmers AJ. Radio resistance of glioma stem cells: Intrinsic characteristic or property of the 'microenvironment-stem cell unit'? Mol Oncol 20115:374-86.

103. Richardson LC, Neri AJ, Tai E, Glenn JD. Testicular cancer: A narrative review of the role of socioeconomic position from risk to survivorship. Urol Oncol 2012;30:95-101.

104. Lo-Coco F, Cicconi L. History of acute promyelocytic leukemia: a tale of endless revolution. Mediterr J Hematol Infect Dis 2011;3:e2011067.

105. Zhou GB, Zhang J, Wang ZY, et al. Treatment of acute promyelocytic leukaemia with all-trans retinoic acid and arsenic trioxide: a paradigm of synergistic molecular targeting therapy. Philos Trans R Soc Lond B Biol Sci 2007; 362:959-71.
106. Nagai S, Takahashi T, Kurokawa M. The impact of molecularly targeted therapies upon the understanding of leukemogenesis and the role of hematopoietic stem cell transplantation in acute promyelocytic leukemia. Curr Stem Cell Res Ther 2010; 5:372-8.

107. Nasr R, de Thé H. Eradication of acute promyelocytic leukemia-initiating cells by PML/RARA-targeting. Int $\mathrm{J}$ Hematol 2010;91:742-7. 